\title{
The influence of incomplete case ascertainment on measures of vaccine efficacy
}

Yue $\mathrm{Wu}^{\mathrm{a}, *}$

yue.wu@telethonkids.org.au

Julie A. Marsh ${ }^{\mathrm{a}, \mathrm{b}}$

Emma S. McBryde

Thomas L. Snelling a, d, e, f

${ }^{a}$ Wesfarmers Centre of Vaccines \& Infectious Diseases, Telethon Kids Institute, University of Western Australia, 100 Roberts Road, Subiaco, Western Australia 6008, Australia

${ }^{\mathbf{b}}$ School of Mathematics \& Statistics, University of Western Australia, 35 Stirling Highway, Crawley, Perth, Western Australia 6009, Australia

${ }^{\mathbf{c}}$ Australian Institute of Tropical Health and Medicine, James Cook University, Townsville, Queensland 4811, Australia

dPrincess Margaret Hospital for Children, Roberts Road, Subiaco, Western Australia 6008, Australia

'Menzies School of Health Research, Charles Darwin University, Casuarina, Northern Territory 0811, Australia

fSchool of Public Health, Curtin University, Kent Street, Bentley, Western Australia 6102, Australia

${ }^{*}$ Corresponding author

\section{Abstract}

\section{Background}

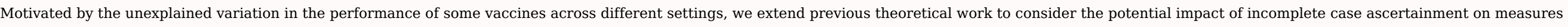

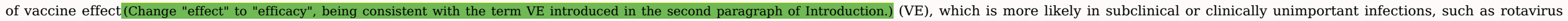
gastroenteritis.

\section{Methods}

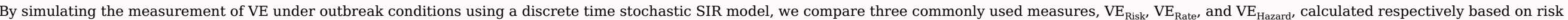

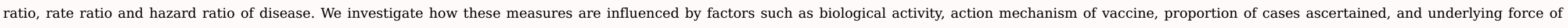
infection.

Results

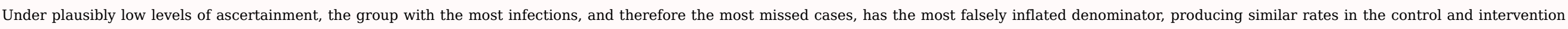

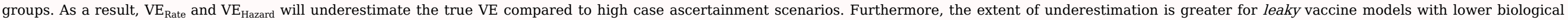
protective effects and under conditions which are conducive to high transmission.

Conclusions

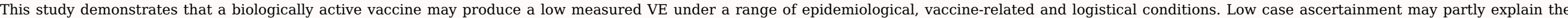
observed heterogeneity in the performance of rotavirus vaccine across different settings, and should be considered in the design and interpretation of future field trials.

Keywords: Vaccine effectiveness; Mathematical modelling; Incomplete ascertainment; Outbreak; Leaky vaccine; All-or-nothing vaccine 


\section{Introduction}

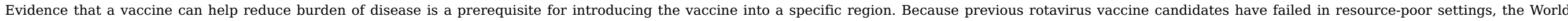

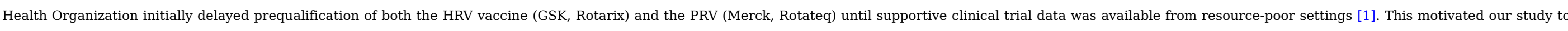
better understand the unexplained variation in the performance of some vaccines across different settings, for which we chose to use rotavirus as an illustrative example.

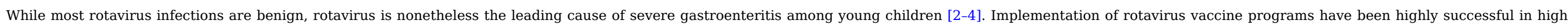

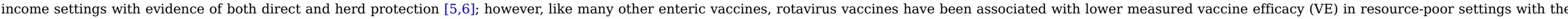

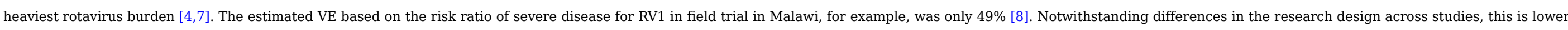

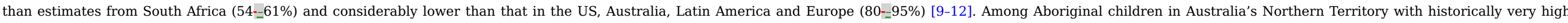
rates of rotavirus infection [13], lower than expected effectiveness was observed during a widespread outbreak [14].

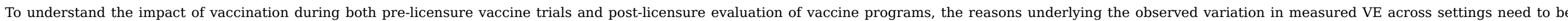

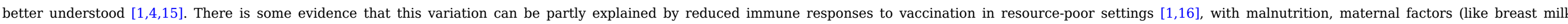

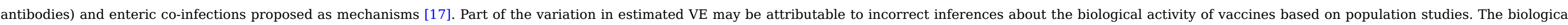

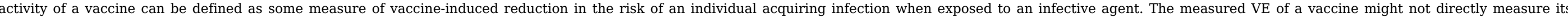

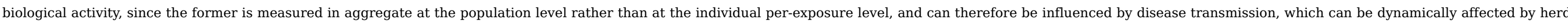
immunity and other population level factors [18].

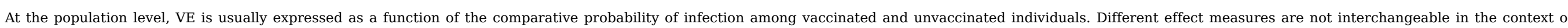

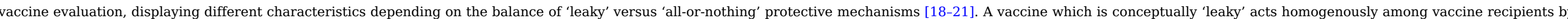

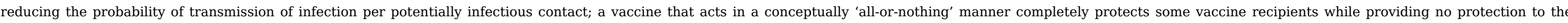

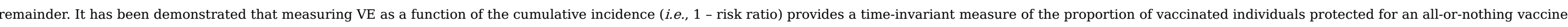

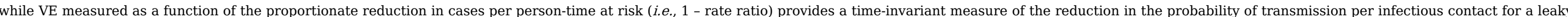
vaccine $[20,22]$.

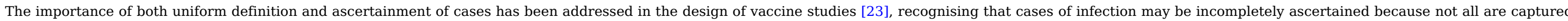

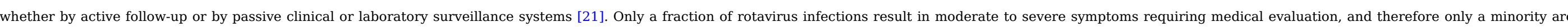

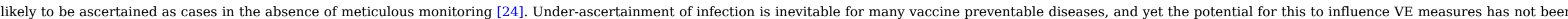
explored. We therefore extend previous theoretical work to consider the potential impact of incomplete ascertainment of infection on the archetypal vaccine models.

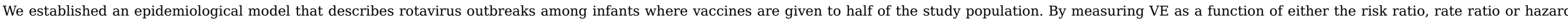

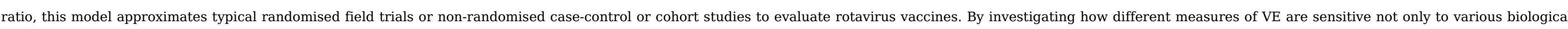

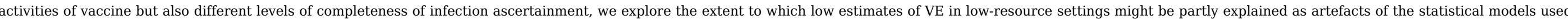
and analytical factors, rather than true differences in the biological activity of the vaccine.

\section{Methods}

\subsection{Stochastic modelling of vaccine trial during rotavirus outbreak}

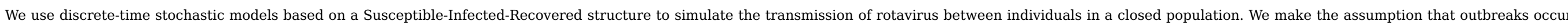

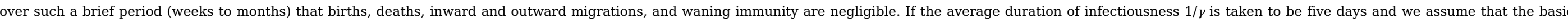

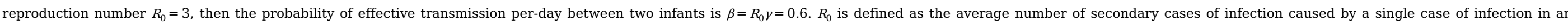

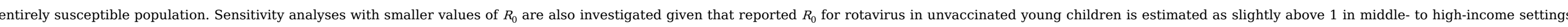




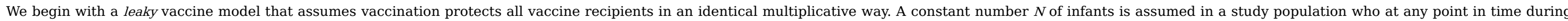

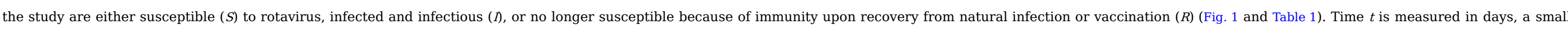

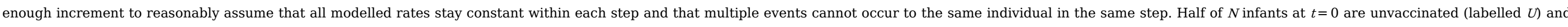

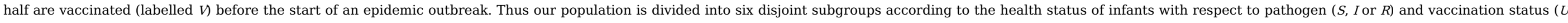

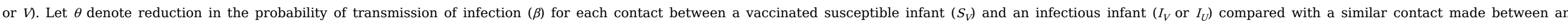
unvaccinated susceptible infant $\left(S_{U}\right)$ and an infectious infant. Let $\Delta R_{U \text { or } V}$ and $\Delta I_{U \text { or } V}$ be the number of newly recovered and infected infants, respectively, during the time interval $\Delta t$ :

$\Delta R_{U} \sim \operatorname{Binomial}\left(I_{U, t}, \gamma \Delta t\right)$
$\Delta R_{V} \sim \operatorname{Binomial}\left(I_{V, t}, \gamma \Delta t\right)$

$\lambda=\beta \frac{I_{U, t}+I_{V, t}}{N}$

$\Delta I_{U} \sim \operatorname{Binomial}\left(S_{U, t}, \lambda \Delta t\right)$

$\Delta I_{V} \sim$ Binomial $\left(S_{V, t},(1-\theta) \lambda \Delta t\right)$

And the size of each subpopulation in the next iteration $t+1$ is,

$S_{U,(t+1)}=S_{U, t}-\Delta I_{U}$

$S_{V,(t+1)}=S_{V, t}-\Delta I_{V}$

$R_{U,(t+1)}=R_{U, t}+\Delta R_{U}$

$R_{V,(t+1)}=R_{V, t}+\Delta R_{V}$

$I_{U,(t+1)}=I_{U, t}+\Delta I_{U}-\Delta R_{U}$

$I_{V,(t+1)}=I_{V, t}+\Delta I_{V}-\Delta R_{V}$

where $\lambda$ represents the force of infection among unvaccinated infants, determined by contact and transmissibility parameter $\beta$ and the proportion of the population being infected at given time $t, \frac{I_{U, t}+I_{V, t}}{N}$.

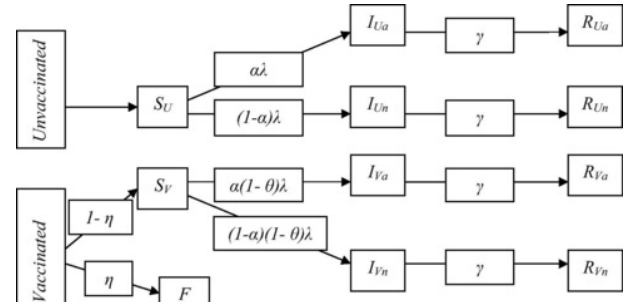

Fig. 1 Model structure. Description and default choice of values of all variables and parameters are provided in Table 1.

Table 1 Variables and parameters.

\begin{tabular}{|c|c|}
\hline Variable & Description \\
\hline$S_{U}$ & Unvaccinated susceptible individuals \\
\hline$S_{V}$ & Vaccinated susceptible individuals \\
\hline$F$ & Fully protected individuals by vaccination \\
\hline$I_{U a}$ & Unvaccinated infections that are ascertained \\
\hline
\end{tabular}




\begin{tabular}{|c|c|c|}
\hline$I_{U n}$ & \multicolumn{2}{|l|}{ Unvaccinated infections that are not ascertained } \\
\hline$I_{V a}$ & \multicolumn{2}{|l|}{ Vaccinated infections that are ascertained } \\
\hline$I_{V n}$ & \multicolumn{2}{|l|}{ Vaccinated infections that are not ascertained } \\
\hline$R_{U a}$ & \multicolumn{2}{|l|}{ Unvaccinated and immunised individuals after recovery from ascertained infections } \\
\hline$R_{U n}$ & \multicolumn{2}{|l|}{ Unvaccinated and immunised individuals after recovery from unascertained infections } \\
\hline$R_{V a}$ & \multicolumn{2}{|l|}{ Vaccinated and immunised individuals after recovery from ascertained infections } \\
\hline$R_{V n}$ & \multicolumn{2}{|l|}{ Vaccinated and immunised individuals after recovery from unascertained infections } \\
\hline Parameter & Description & Default value by scenario \\
\hline$N$ & Total size of the study population & 10,000 \\
\hline$\beta$ & Transmission probability per-infectious-contact & 0.6 \\
\hline$\gamma$ & Rate of recovery & $0.2, R_{0}=\beta / \gamma=3$ \\
\hline$\theta$ & $\begin{array}{l}\text { Biological activity of a leaky vaccine: reduction in the probability of transmission of infection for each contact between a vaccinated and an } \\
\text { infectious individual }\end{array}$ & $\begin{array}{l}0.8 \text { for a leaky vaccine, and } 0 \text { for an all-or-nothing } \\
\text { vaccine }\end{array}$ \\
\hline$\eta$ & Biological activity of an all-or-nothing vaccine: proportion fully protected by vaccination & $\begin{array}{l}0 \text { for a leaky vaccine, and } 0.8 \text { for an all-or-nothing } \\
\text { vaccine. }\end{array}$ \\
\hline$\alpha$ & Proportion of infection ascertained & 0.1 \\
\hline
\end{tabular}

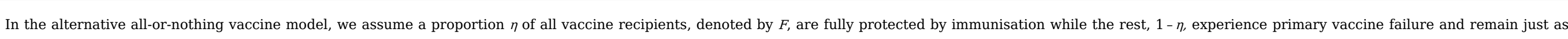

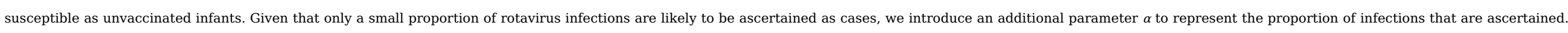
Ascertained and unascertained cases are denoted by subscripts $a$ and $n$, respectively.

\subsection{Measures of vaccine effect}

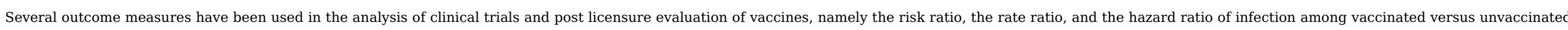

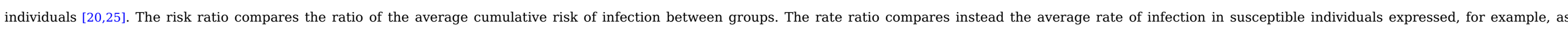

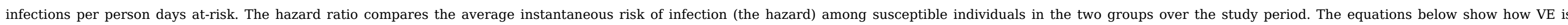
calculated as a function of these distinct measures at the end of an outbreak $t=T$ :

$\mathrm{VE}_{\text {Risk }}=1-$ risk ratio $=1-\frac{R_{V a, T}}{R_{U a, T}}$

$\mathrm{VE}_{\text {Rate }}=1-$ rate ratio $=1-\frac{R_{V a, T} / \sum_{t=1}^{t=T}\left[S_{V, t}+F_{t}+R_{V n, t}\right]}{R_{U a, T} / \sum_{t=1}^{t=T}\left[S_{U, t}+R_{U n, t}\right]} ;$

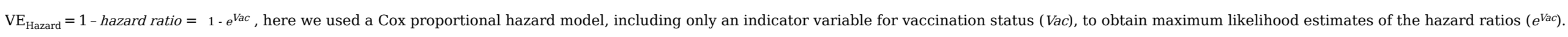

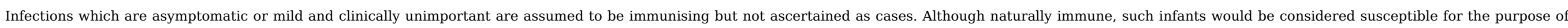
calculating the VE measures.

\subsection{Simulation and output variables}




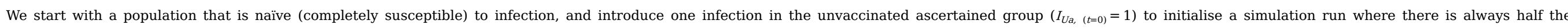

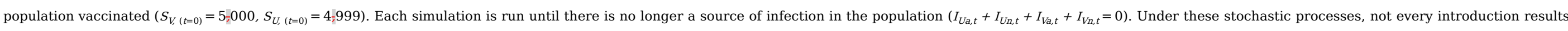

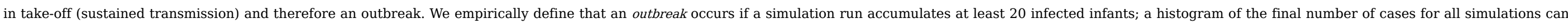

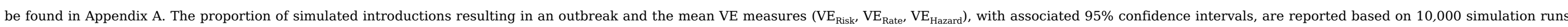
under default parameter values (Table 1) unless specified otherwise. All simulations and analyses were conducted in R [26].

\section{Results}

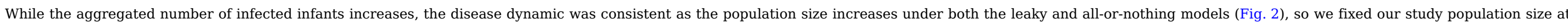

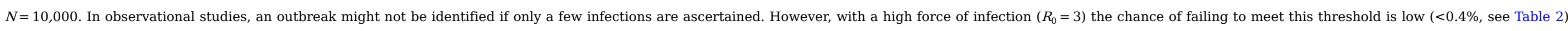
even if the proportion of infections ascertained is low.
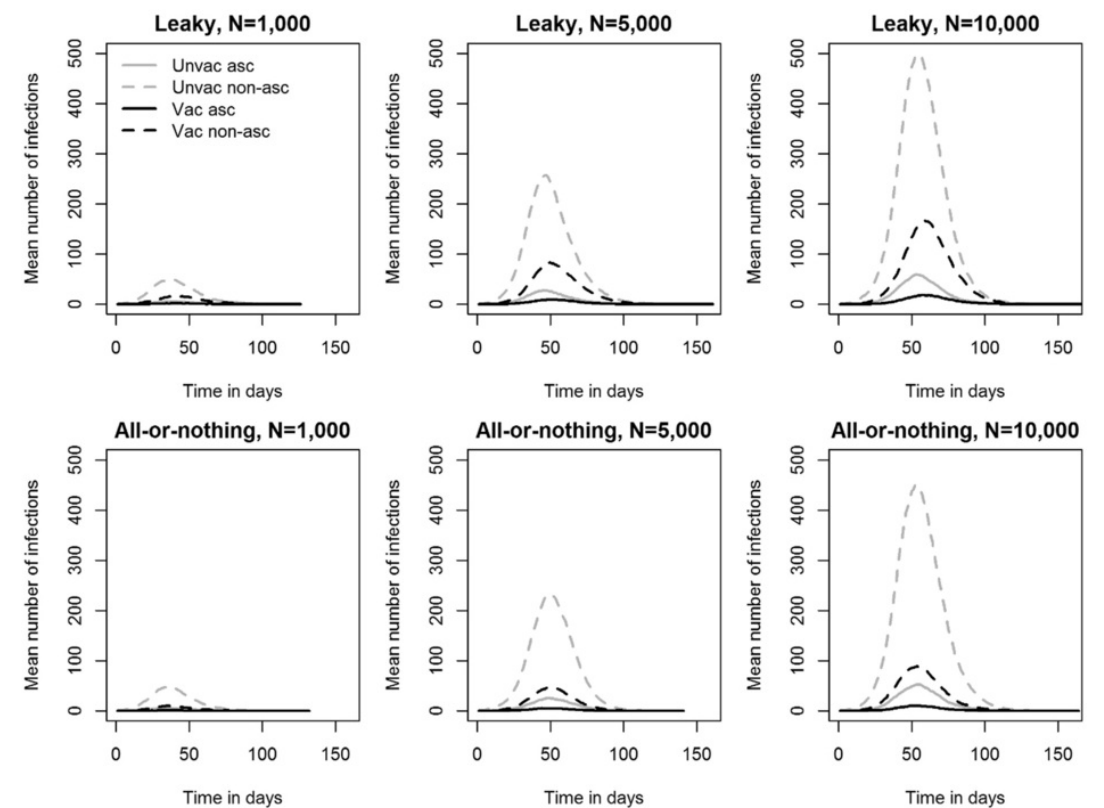

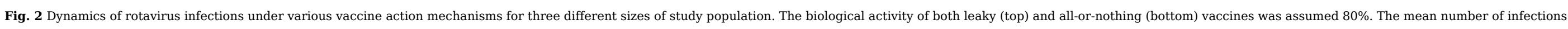

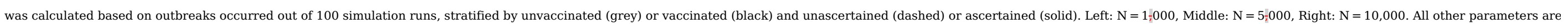
as given in Table 1 .

Table 2 Mean measures of VE based on default parameters as given in Table 1 unless specified otherwise, 10,000 simulation runs.

\begin{tabular}{|c|c|c|c|c|}
\hline VE measures $\backslash$ Scenario & $\begin{array}{c}\text { Leaky, } \\
\alpha=1[95 \mathrm{CI}]\end{array}$ & $\begin{array}{l}\text { All-or-nothing, } \\
\alpha=1 \text { [95CI] }\end{array}$ & $\begin{array}{c}\text { Leaky, } \\
\alpha=0.1[95 \mathrm{CI}]\end{array}$ & $\begin{array}{l}\text { All-or-nothing, } \\
\alpha=0.1 \text { [95CI] }\end{array}$ \\
\hline Risk of outbreaks & 0.4925 & 0.4929 & 0.4968 & 0.4956 \\
\hline Ascertained outbreaks & 0.4925 & 0.4929 & 0.494 & 0.4918 \\
\hline $\mathrm{VE}_{\text {Risk }}$ & $0.6557[0.6554,0.6560]$ & $0.8001[0.8000,0.8002]$ & $0.6550[0.6545,0.6555]$ & $\begin{array}{l}0.7996 \\
{[0.7992,0.7999]}\end{array}$ \\
\hline
\end{tabular}




\begin{tabular}{|l|l|l|l|}
\hline $\mathrm{VE}_{\text {Rate }}$ & $0.8352[0.8350,0.8354]$ & $0.9023[0.9021,0.9025]$ & $0.6734[0.6730,0.6739]$ \\
\hline $\mathrm{VE}_{\text {Hazard }}$ & $0.8011[0.8003,0.8018]$ & $0.8758[0.8753,0.8762]$ & 0.8113 \\
{$[0.8110,0.8116]$}
\end{tabular}

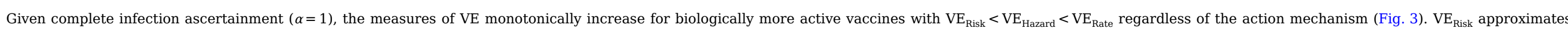

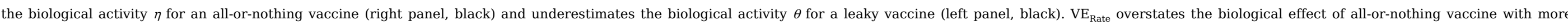

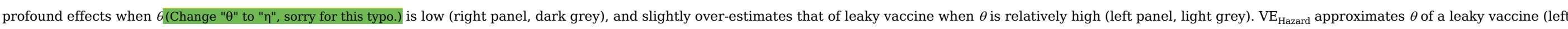

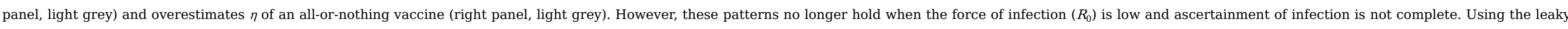

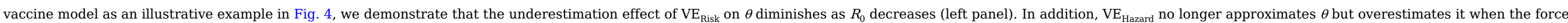

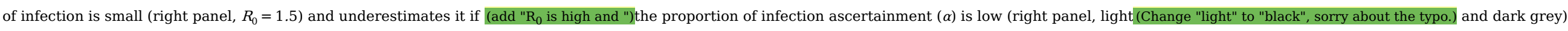
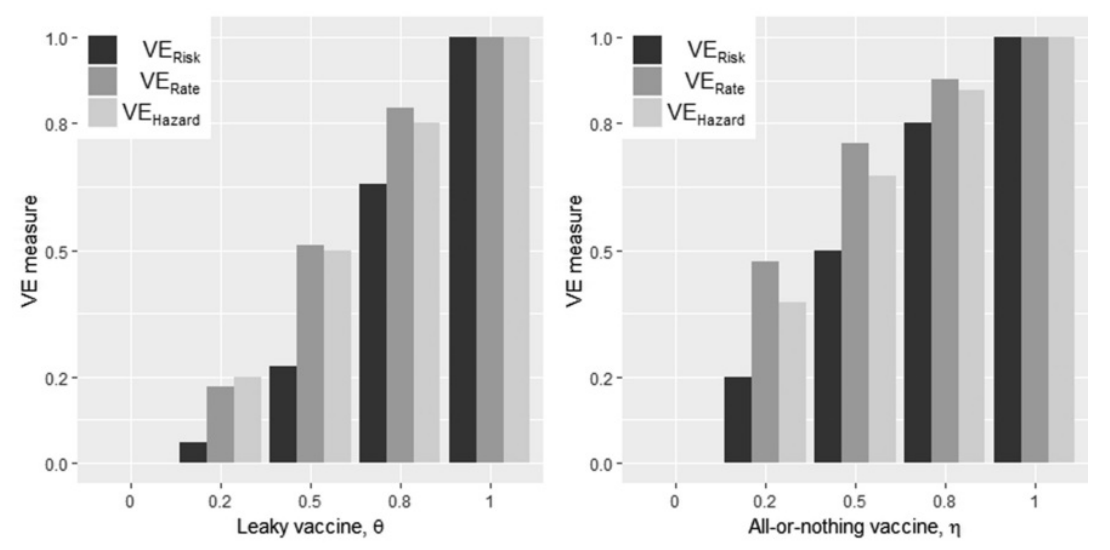

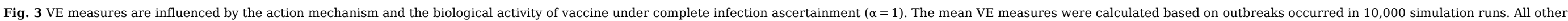
parameters are as given in Table 1.

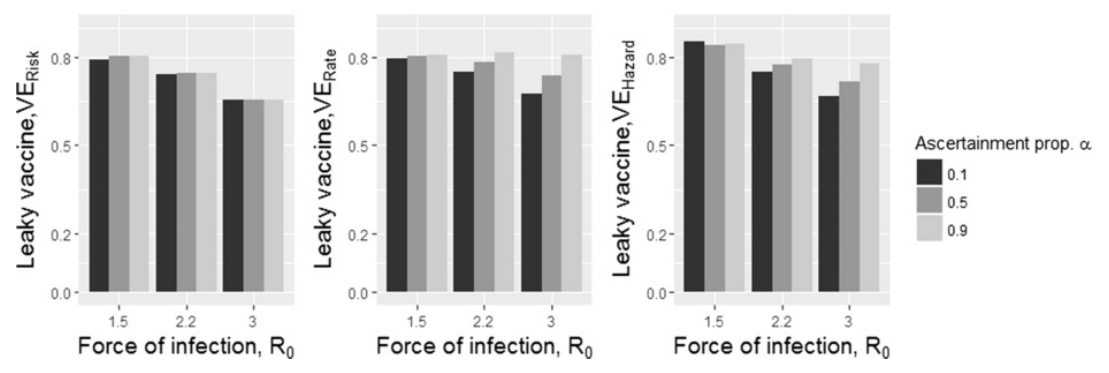

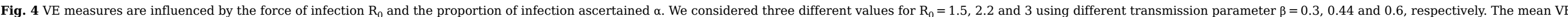
measures were calculated based on outbreaks occurred in 10,000 simulation runs. The biological activity of the leaky vaccine was assumed $80 \%$. All other parameters are as given in Table 1 .

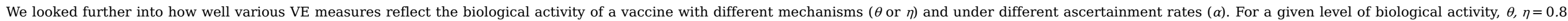

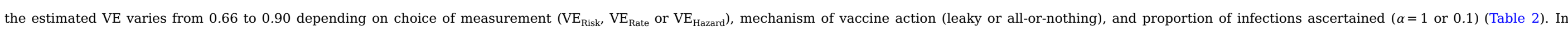

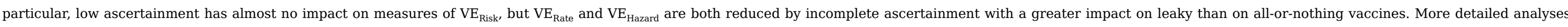
are included in Appendix B. Epidemiological factors and logistical factors interactively contribute to estimates of VE. 


\section{Discussion}

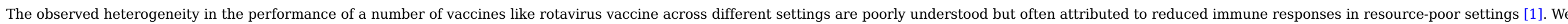

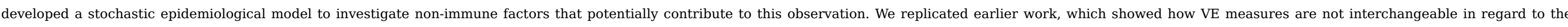

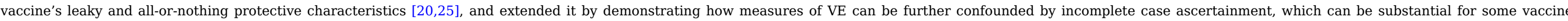

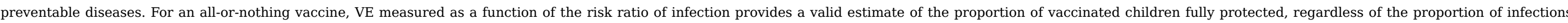

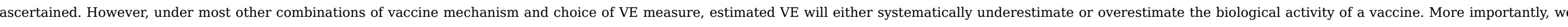

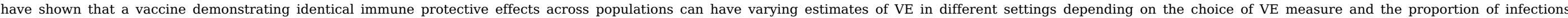
ascertained.

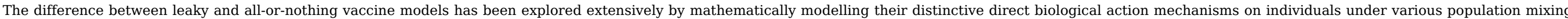

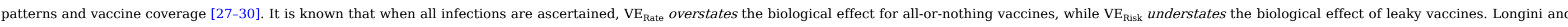

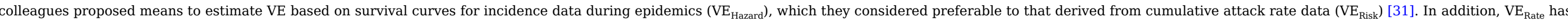

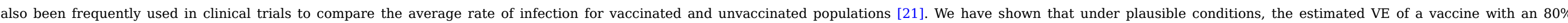

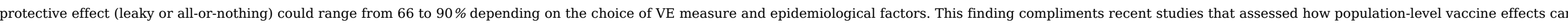
be influenced by a range of disease and vaccine-related factors [16,18].

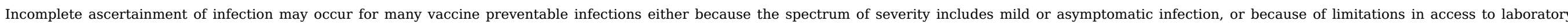

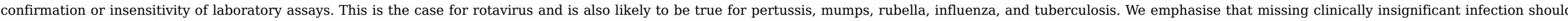

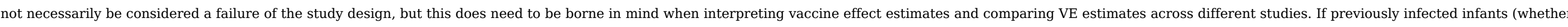

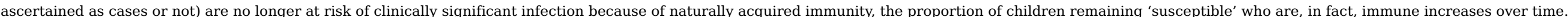

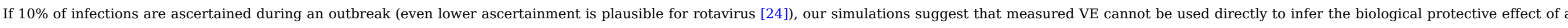

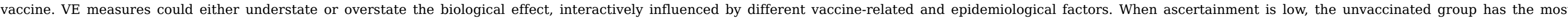

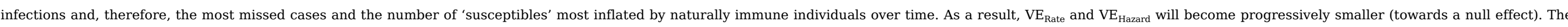

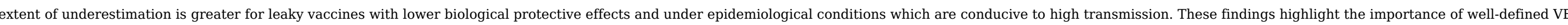

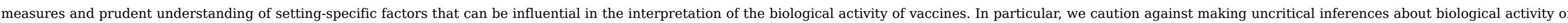
vaccines (including of waning efficacy) on the basis of population-based effect measures.

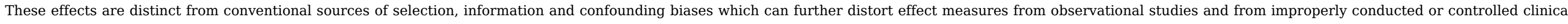

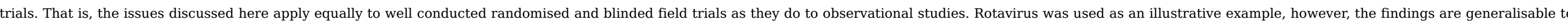

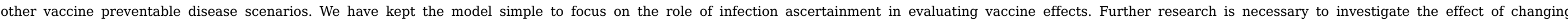

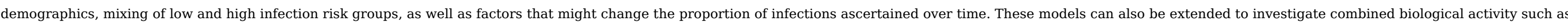
vaccines with combined leaky and all-or-nothing effects.

\section{Conclusion}

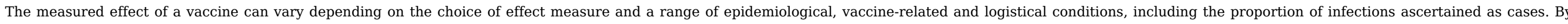

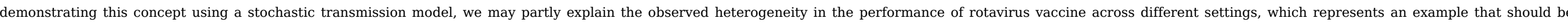
generalisable to many other infectious disease scenarios. This work should guide the design and interpretation of future vaccine trials.

\section{Conflicts of interests}

None declared. 


\section{Author contributions}

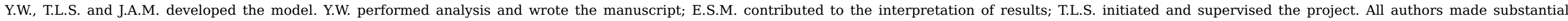
contribution to manuscript writing and approved the submission of the manuscript.

\section{Acknowledgement}

This work was funded by Telethon Kids Institute. T.L.S. was supported by a National Health and Medical Research Council fellowship [CDF1111657].

\section{Appendix A. Supplementary material}

Supplementary data associated with this article can be found, in the online version, at https://doi.org/10.1016/j.vaccine.2018.04.046.

\section{References}

[1] V. Jiang, B. Jiang, J. Tate, U.D. Parashar and M.M. Patel, Performance of rotavirus vaccines in developed and developing countries, Human Vaccines 6 (7), 2010 , 532-542.

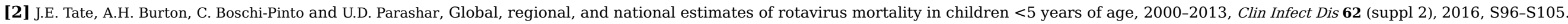

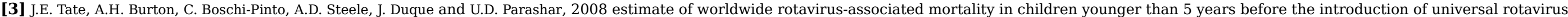
vaccination programmes: a systematic review and meta-analysis, Lancet Infect Dis 12 (2), 2012, 136-141.

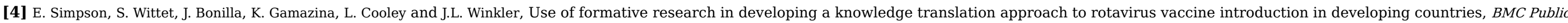
Health 7 (1), 2007, 281.

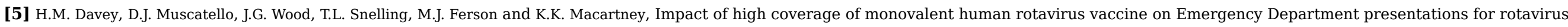
gastroenteritis, Vaccine 33 (14), 2015, 1726-1730.

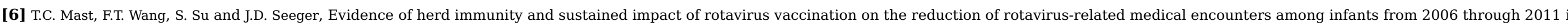
the United States, Pediatr Infect Dis J 34 (6), 2015, 615-620.

[7] J.S. Bresee, U.D. Parashar, M.-A. Widdowson, J.R. Gentsch, A.D. Steele and R.I. Glass, Update on rotavirus vaccines, Pediatr Infect Dis J 24 (11), 2005 , $947-952$.

[8] S.A. Madhi, N.A. Cunliffe, D. Steele, D. Witte, M. Kirsten, C. Louw, et al., Effect of human rotavirus vaccine on severe diarrhea in african infants, $N$ Engl J Med 362 (4), $2010,289-298$.

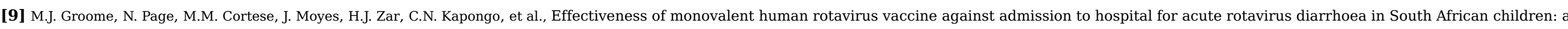
case-control study, Lancet Infect Dis 14 (11), 2014, 1096-1104.

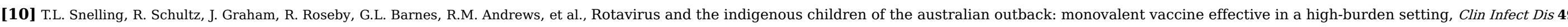
(3), 2009, 428-431.

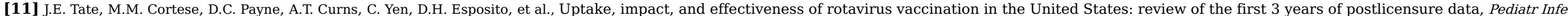
Dis J 30 (1), 2011, S56-S60.

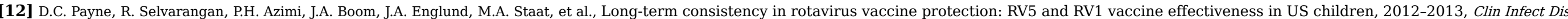
2015.

[13] A.T. Newall, R. MacIntyre, H. Wang, B. Hull and K. Macartney, Burden of severe rotavirus disease in Australia, J Paediatr Child Health 42 (9), $2006,521-527$.

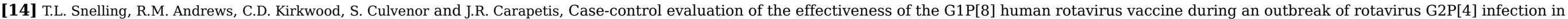
Central Australia, Clin Infect Dis 52 (2), 2011, 191-199. 
[15] K. Grimwood and S.B. Lambert, Rotavirus vaccines: opportunities and challenges, Human Vaccines 5 (2), 2009, 57-69.

[16] B.A. Lopman, V.E. Pitzer, R. Sarkar, B. Gladstone, M. Patel, J. Glasser, et al., Understanding reduced rotavirus vaccine efficacy in low socio-economic settings, PLOS One 7 (8), 2012, e41720.

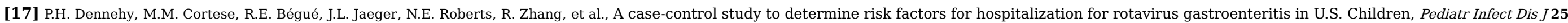
(12), 2006, 1123-1131.

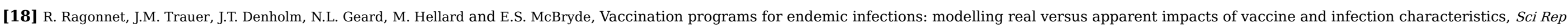
5, 2015, 15468.

[19] M.E. Halloran, Overview of vaccine field studies: types of effects and designs, J Biopharm Stat 16 (4), 2006, 415-427.

[20] P. Smith, L. Rodrigues and P. Fine, Assessment of the protective efficacy of vaccines against common diseases using case-control and cohort studies, Int J Epidemiol 13 (1), 1984, 87-93.

[21] M.E. Halloran, I.M. Longini and C.J. Struchiner, Design and analysis of vaccine studies, 2010, Springer; New York.

[22] S. Greenland and R. Frerichs, On measures and models for the effectiveness of vaccines and vaccination programmes, Int J Epidemiol 17 (2), $1988,456-463$.

[23] W. Orenstein, R. Bernier, T. Dondero, A. Hinman, J. Marks, K. Bart, et al., Field evaluation of vaccine efficacy, Bull World Health Organ 63 (6), 1985, $1055-1068$.

[24] G. Chowell, Mathematical and statistical estimation approaches in epidemiology, 2009, Springer; Dordrecht.

[25] R.C. Brunet, C.J. Struchiner and M.E. Halloran, On the distribution of vaccine protection under heterogeneous response, Math Biosci 116 (1), $1993,111-125$.

[26] R Core Team. R: A Language and Environment for Statistical Computing. Vienna, Austria: R Foundation for Statistical Computing; 2014.

[27] M.E. Halloran, M. Haber and I.M. Longini, Interpretation and estimation of vaccine efficacy under heterogeneity, Am J Epidemiol 136 (3), 1992 , $328-343$.

[28] I.M. Longini, M.E. Halloran and M. Haber, Estimation of vaccine efficacy from epidemics of acute infectious agents under vaccine-related heterogeneity, Math Biosci 117 (1), $1993,271-281$.

[29] I.M. Longini, M.E. Halloran, M. Haber and R.T. Chen, Measuring vaccine efficacy from epidemics of acute infectious agents, Stat Med 12 (3-4), $1993,249-263$.

[30] M.S. Rahman, Estimating vaccine efficacy under the heterogeneity of vaccine action in a nonrandomly mixing population, J Biopharm Stat 23 (2), 2013 , 394-412.

[31] I.M. Longini and M.E. Halloran, A frailty mixture model for estimating vaccine efficacy, J Roy Stat Soc: Ser C (Appl Stat) 45 (2), 1996, 165-173.

\section{Appendix A. Supplementary material}

Multimedia Component 1

Supplementary Figs. S1-S4

\section{Queries and Answers}

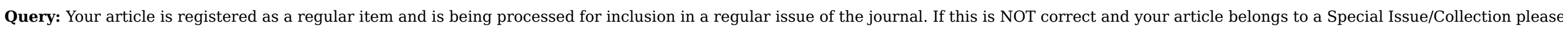
contact jd.bailey@elsevier.com immediately prior to returning your corrections.

Answer: Yes

Query: The author names have been tagged as given names and surnames (surnames are highlighted in teal color). Please confirm if they have been identified correctly.

Answer: Yes

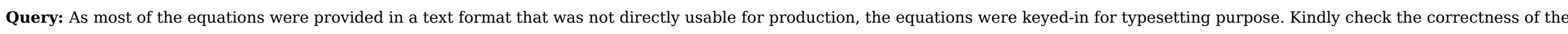
equations and amend wherever required. 


\section{Answer: Yes}

